\title{
New angucycline $C$-glycosides from Streptomyces sp. RI33
}

\author{
Jun-ya Ueda ${ }^{1}$, Miho Izumikawa ${ }^{1}$, Akira Mukai ${ }^{1}$, Aya Nagai ${ }^{1}$, Ji-Hwan Hwang ${ }^{1}$, Motoki Takagi ${ }^{1}$ \\ and Kazuo Shin-ya ${ }^{2}$
}

In the course of our screening program for anticancer agents from natural sources, five new angucyclines, JBIR-90 (1), -116 (2), -91 (3), -92 (4) and -93 (5), were isolated from the fermentation broth of Streptomyces sp. RI33. The structures of $1-5$ were elucidated on the basis of 1D and 2D NMR spectroscopy and MS analyses. Compounds 2-4 showed cytotoxic activities against malignant pleural mesothelioma cells at $\mathrm{IC}_{50}$ values of $20-50 \mu \mathrm{m}$.

The Journal of Antibiotics (2011) 64, 367-372; doi:10.1038/ja.2011.8; published online 16 February 2011

Keywords: angucycline; cytotoxicity; malignant pleural mesothelioma; Streptomyces; tetraphene quinoid; urdamycin

\section{INTRODUCTION}

Malignant pleural mesothelioma (MPM), which is associated with exposure to asbestos fibers, is an aggressive neoplasm that develops from the pleura and is highly invasive to surrounding tissues. ${ }^{1,2}$ MPM has been shown to be resistant to all conventional therapies, including chemotherapy, radiotherapy and surgery, and the prognosis of patients remains very poor. Consequently, it is a tumor that continues to be a difficult clinical problem. ${ }^{3-5}$ Therefore, developing novel therapeutic agents against MPM cells are strongly desired. We have already reported that a novel compound, JBIR-23, which was isolated from Streptomyces sp. AK-AB27, showed antitumor activity against MPM cells, with tubulin polymerization-promoting activity. ${ }^{6,7}$ We have also discovered novel anti-MPM compounds, namely, the teleocidin analog JBIR- $31,{ }^{8}$ the aminocaprophenone alkaloid ficuseptamine $\mathrm{B}^{9}$ and the 1,1-dichlorocyclopropane-skeleton-containing angucycline JBIR- $88 .{ }^{10}$ In the course of our screening program for cytotoxic compounds against MPM cells from microbial metabolites, we succeeded in isolating new compounds termed JBIR-90 (1), -116 (2), -91 (3), -92 (4) and -93 (5) from the culture of Streptomyces sp. RI33 (Figure 1). This paper describes the fermentation, isolation, structure elucidation and, in brief, the biological activity of 1-5.

\section{RESULTS AND DISCUSSION}

Structure elucidation of 1

Compound 1 was obtained as a yellow powder. The IR spectrum of 1 revealed the characteristic absorptions of a ketone $\left(v_{\max } 1705 \mathrm{~cm}^{-1}\right)$ and a quinone $\left(v_{\max } 1620 \mathrm{~cm}^{-1}\right)$ group. The molecular formula of 1 was determined to be $\mathrm{C}_{25} \mathrm{H}_{24} \mathrm{O}_{7}$ on the basis of HR-ESI-MS data. The direct connectivity between each proton and carbon was established by the heteronuclear single-quantum coherence spectrum, and the ${ }^{13} \mathrm{C}$ and ${ }^{1} \mathrm{H}$ NMR spectral data for $\mathbf{1}$ are shown in Table 1 . The analyses of double-quantum-filtered (DQF)-COSY and constant time heteronuclear multiple bond correlation $(\mathrm{HMBC})^{11}$ spectra revealed the structure of 1 as follows.

The sequence from methylene protons $2-\mathrm{H}\left(\delta_{\mathrm{H}} 3.02,2.56\right)$ to methylene protons $4-\mathrm{H}\left(\delta_{\mathrm{H}} 2.98,2.67\right)$ through a methine proton $3-\mathrm{H}\left(\delta_{\mathrm{H}} 2.46\right)$, which was in turn coupled to a methyl proton $3-\mathrm{CH}_{3}$ $\left(\delta_{\mathrm{H}} 1.20\right)$, was observed in the DQF-COSY (Figure 2$)$. The HMBC correlations from $2-\mathrm{H}$ to an $\alpha, \beta$-unsaturated ketone carbonyl carbon $\mathrm{C}-1\left(\delta_{\mathrm{C}} 199.3\right)$ and an aromatic carbon C-12b $\left(\delta_{\mathrm{C}} 136.6\right)$, and from $4-$ $\mathrm{H}$ to two aromatic carbons, $\mathrm{C}-4 \mathrm{a}\left(\delta_{\mathrm{C}} 150.4\right)$ and $\mathrm{C}-12 \mathrm{~b}$, established a 3-methylhex-5-en-1-one substructure. The spin coupling between two aromatic protons, $5-\mathrm{H}\left(\delta_{\mathrm{H}} 7.53\right)$ and $6-\mathrm{H}\left(\delta_{\mathrm{H}} 8.28\right)$, together with ${ }^{1} \mathrm{H}-{ }^{13} \mathrm{C}$ long-range couplings from $5-\mathrm{H}$ to two aromatic carbons, C-6a $\left(\delta_{\mathrm{C}} 133.5\right)$ and $\mathrm{C}-12 \mathrm{~b}$, and from $6-\mathrm{H}$ to two aromatic carbons, $\mathrm{C}-4 \mathrm{a}$ and $\mathrm{C}-12 \mathrm{a}\left(\delta_{\mathrm{C}} 135.9\right)$, indicated a 3-methyl-1-oxo-1,2,3,4-tetrahydronaphthalene structure. An aromatic proton $11-\mathrm{H}\left(\delta_{\mathrm{H}} 7.71\right)$, which was spin-coupled with another aromatic proton $10-\mathrm{H}\left(\delta_{\mathrm{H}} 7.90\right)$, was strongly meta-coupled to two aromatic carbons, C-7a $\left(\delta_{\mathrm{C}} 114.8\right)$ and $\mathrm{C}-9\left(\delta_{\mathrm{C}} 137.1\right)$, and long-range coupled to an aromatic carbon C-11a $\left(\delta_{\mathrm{C}} 133.7\right)$, a quinone carbonyl carbon C-12 $\left(\delta_{\mathrm{C}} 182.8\right)$ located at the peri-position, and another quinone carbonyl carbon C-7 $\left(\delta_{\mathrm{C}}\right.$ 188.0). The aromatic proton $10-\mathrm{H}$ was meta-coupled to two aromatic carbons, C-8 $\left(\delta_{\mathrm{C}} 158.2\right)$ and C-11a, and W-letter long-range-coupled to a quinone carbonyl carbon $\mathrm{C}-12$. The long-range coupling from a hydrogen-bonded phenolic hydroxyl proton $8-\mathrm{OH}\left(\delta_{\mathrm{H}} 12.72\right)$ to three

${ }^{1}$ Biomedicinal Information Research Center (BIRC), Japan Biological Informatics Consortium (JBIC), Tokyo, Japan and ${ }^{2}$ Biomedicinal Information Research Center (BIRC), National Institute of Advanced Industrial Science and Technology (AIST), Tokyo, Japan

Correspondence: Dr M Takagi, Biomedicinal Information Research Center (BIRC), Japan Biological Informatics Consortium (JBIC), 2-4-7 Aomi, Koto-ku, Tokyo 135-0064, Japan. E-mail: motoki-takagi@aist.go.jp or

Dr K Shin-ya, Biomedicinal Information Research Center (BIRC), National Institute of Advanced Industrial Science and Technology (AIST), 2-4-7 Aomi, Koto-ku, Tokyo 135-0064, Japan.

E-mail: k-shinya@aist.go.jp

Received 1 December 2010; revised 19 January 2011; accepted 23 January 2011; published online 16 February 2011 
<smiles>CC1CC(=O)c2c(ccc3c2C(=O)c2ccc(C4(C)CC5CC4OC(C)(C)C5)c(O)c2C3=O)C1</smiles>

JBIR-90 (1)

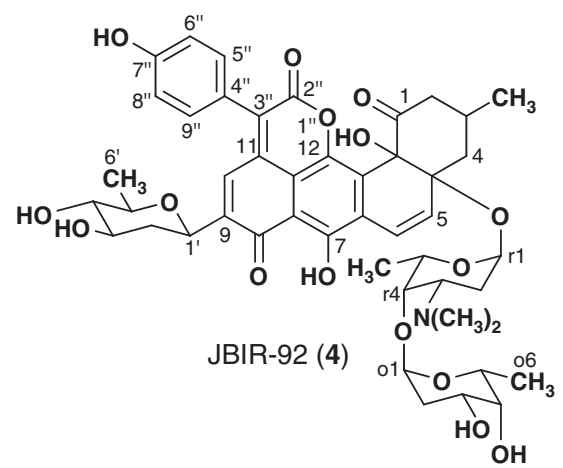





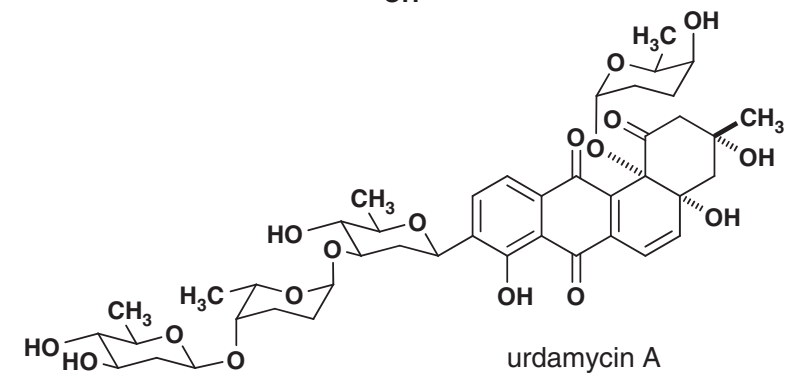

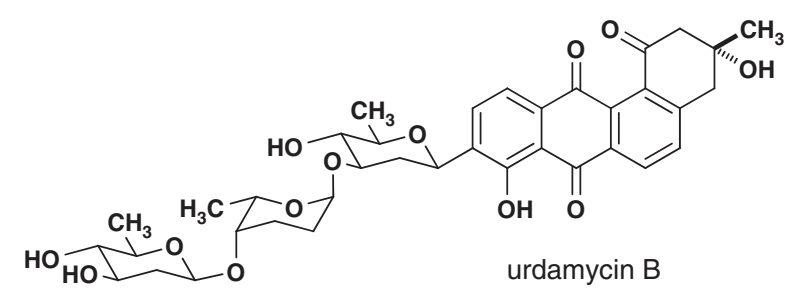

Figure 1 Structures of $\mathbf{1 - 5}$

aromatic carbons, C-7a, C-8 and C-9, established a naphthoquinone substructure. The connectivity between these two substructures was established by the long-range couplings from $5-\mathrm{H}$ to the quinone carbonyl carbon C-7, which suggested a 1,2,3,4-tetrahydrotetraphene skeleton. This chromophore moiety was also supported by the UV absorptions ( $\lambda_{\max } 268$ and $405 \mathrm{~nm}$ ), which corresponded to those of urdamycin $\mathrm{B}^{12,13}$ and urdamycinone B. ${ }^{14}$

A remaining pseudo-glycoside unit was established by the sequence from an oxymethine proton $1^{\prime}-\mathrm{H}\left(\delta_{\mathrm{H}} 5.19\right)$ to a methyl proton $6^{\prime}-\mathrm{H}\left(\delta_{\mathrm{H}} 1.37\right)$ through two methylene protons, $2^{\prime}-\mathrm{H}\left(\delta_{\mathrm{H}} 2.44\right.$, $1.40)$, and three oxymethine protons, $3^{\prime}-\mathrm{H}\left(\delta_{\mathrm{H}} 4.03\right), 4^{\prime}-\mathrm{H}\left(\delta_{\mathrm{H}}\right.$ 3.76) and $5^{\prime}-\mathrm{H}\left(\delta_{\mathrm{H}} 4.44\right)$, observed in the DQF-COSY, and a longrange coupling between $1^{\prime}-\mathrm{H}$ and $\mathrm{C}-5^{\prime}\left(\delta_{\mathrm{C}} 72.6\right)$. The large coupling constants between $1^{\prime}-\mathrm{H} / 2^{\prime}-\mathrm{H}_{\mathrm{ax}}\left(J_{1^{\prime}-\mathrm{H}-2^{\prime}-\mathrm{Hax}} \sim 12.0 \mathrm{~Hz}\right), 2^{\prime}-\mathrm{H}_{\mathrm{ax}} / 3^{\prime}-\mathrm{H}$ $\left(J_{2^{\prime}-\mathrm{Hax}-3^{\prime}-\mathrm{H}} \sim 11.5 \mathrm{~Hz}\right), 3^{\prime}-\mathrm{H} / 4^{\prime}-\mathrm{H}\left(J_{3^{\prime}-\mathrm{H}-4^{\prime}-\mathrm{H}} \sim 9.0 \mathrm{~Hz}\right)$ and $4^{\prime}-\mathrm{H} / 5^{\prime}-\mathrm{H}$

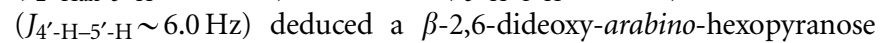
(olivopyranose) structure. The high-field shift at the anomeric carbon
$\mathrm{C}-1^{\prime}\left(\delta_{\mathrm{C}} 64.3\right)$, compared with those of usual anomeric carbon shifts, and the HMBC correlations from $1^{\prime}-\mathrm{H}$ to three aromatic carbons, $\mathrm{C}-8$, C-9 and C-10 $\left(\delta_{\mathrm{C}} 133.7\right)$, indicated that the saccharide unit directly attached at C-9. Thus, the structure of $\mathbf{1}$ was determined as an angucycline $C$-olivopyranoside, 3-deoxyurdamycinone $\mathrm{B}$, as shown in Figure 1.

\section{Structure elucidation of 2}

Compound 2 was obtained as a dark yellow powder. The IR spectrum of 2 revealed the characteristic absorptions of a ketone ( $v_{\max }$ $\left.1720 \mathrm{~cm}^{-1}\right)$ and a quinone $\left(v_{\max } 1640 \mathrm{~cm}^{-1}\right)$ group. Its molecular formula was determined as $\mathrm{C}_{37} \mathrm{H}_{46} \mathrm{O}_{15}$ by analysis of the HR-ESI-MS spectrum. The ${ }^{13} \mathrm{C}$ and ${ }^{1} \mathrm{H}$ NMR spectral data for 2 are summarized in Table 1.

The sequence from methylene protons $2-\mathrm{H}\left(\delta_{\mathrm{H}} 2.60,2.25\right)$ to methylene protons $4-\mathrm{H}\left(\delta_{\mathrm{H}} 1.98,1.56\right)$ through a methine proton $3-\mathrm{H}\left(\delta_{\mathrm{H}} 2.24\right)$, which was in turn coupled to a methyl proton $3-\mathrm{CH}_{3}$ 
Table $1{ }^{13} \mathrm{C}$ and ${ }^{1} \mathrm{H}$ NMR data for $1-5$

\begin{tabular}{|c|c|c|c|c|c|c|c|c|c|c|}
\hline \multirow[b]{2}{*}{ Position } & \multicolumn{2}{|r|}{$1^{a, d}$} & \multicolumn{2}{|r|}{$2^{b, e}$} & \multicolumn{2}{|r|}{$3^{b, d}$} & \multicolumn{2}{|r|}{$4^{b, e}$} & \multicolumn{2}{|r|}{$\mathbf{5}^{c, e}$} \\
\hline & $\delta_{C}$ & $\begin{array}{l}\delta_{H} \\
\text { (multiplicity, } \\
\mathrm{J} \text { in } \mathrm{Hz} \text { ) }\end{array}$ & $\delta_{C}$ & $\begin{array}{l}\delta_{H} \\
\text { (multiplicity, } \\
\mathrm{J} \text { in } \mathrm{Hz} \text { ) }\end{array}$ & $\delta_{C}$ & $\begin{array}{l}\delta_{H} \\
\text { (multiplicity, } \\
\mathrm{J} \text { in } \mathrm{Hz} \text { ) }\end{array}$ & $\delta_{C}$ & $\begin{array}{l}\delta_{H} \\
\text { (multiplicity, } \\
\mathrm{J} \text { in } \mathrm{Hz} \text { ) }\end{array}$ & $\delta_{C}$ & $\begin{array}{l}\delta_{H} \\
\text { (multiplicity, } \\
\mathrm{J} \text { in } \mathrm{Hz} \text { ) }\end{array}$ \\
\hline 1 & 199.3 & & 208.2 & & 208.5 & & 209.1 & & 208.0 & \\
\hline 2 & 47.5 & $\begin{array}{l}3.02(\mathrm{ddd}, 16.0,5.5,1.5) \\
2.56(\mathrm{dd}, 16.0,11.0)\end{array}$ & 47.8 & $\begin{array}{l}2.60(\mathrm{dd}, 8.0,2.0) \\
2.25(\mathrm{~m})\end{array}$ & 48.1 & $\begin{array}{l}2.58(\mathrm{dd}, 8.5,2.0) \\
2.29(\mathrm{~m})\end{array}$ & 47.5 & $\begin{array}{l}2.72(\mathrm{dt}, 12.5,3.5) \\
2.52(\mathrm{t}, 12.5)\end{array}$ & 46.7 & $\begin{array}{l}2.66(\mathrm{dt}, 12.5,3.0) \\
2.58(\mathrm{t}, 12.5)\end{array}$ \\
\hline 3 & 30.7 & 2.46 (br sept-like, $\sim 5.5$ ) & 32.3 & $2.24(\mathrm{~m})$ & 32.3 & $2.30(\mathrm{~m})$ & 31.1 & $2.32(\mathrm{~m})$ & 30.7 & $2.31(\mathrm{~m})$ \\
\hline $3-\mathrm{CH}_{3}$ & 21.5 & $1.20(\mathrm{~d}, 6.5)$ & 21.8 & $1.01(\mathrm{~d}, 5.0)$ & 21.8 & $1.02(\mathrm{~d}, 6.0)$ & 21.9 & $0.99(d, 6.5)$ & 21.7 & $0.92(d, 6.5)$ \\
\hline 4 & 38.4 & $\begin{array}{l}2.98(\mathrm{dd}, 16.5,1.5) \\
2.67(\mathrm{dd}, 16.5,11.0)\end{array}$ & 44.3 & $\begin{array}{l}1.98(\mathrm{dd}, 13.5,2.0) \\
1.56(\mathrm{dd}, 13.5,11.5)\end{array}$ & 44.3 & $\begin{array}{l}1.99(\mathrm{~m}) \\
1.63(\mathrm{dd}, 14.0,11.5)\end{array}$ & 44.8 & $\begin{array}{l}2.01(\mathrm{dt}, 14.0,2.0) \\
1.56(\mathrm{dd}, 14.0,12.5)\end{array}$ & 44.3 & $\begin{array}{l}2.00(\mathrm{dd}, 14.0,2.0) \\
1.57(\mathrm{dd}, 14.0,12.5)\end{array}$ \\
\hline $4 a$ & 150.4 & & 87.6 & & 87.7 & & 86.5 & & 85.7 & \\
\hline 5 & 133.0 & $7.53(d, 8.0)$ & 142.7 & $6.95(d, 10.0)$ & 142.1 & $7.00(\mathrm{~d}, 10.0)$ & 134.6 & $6.76(d, 10.0)$ & 134.1 & $6.80(d, 10.0)$ \\
\hline 6 & 128.9 & $8.28(d, 8.0)$ & 119.7 & $7.10(\mathrm{~d}, 10.0)$ & 120.3 & $7.06(d, 10.0)$ & 121.3 & $7.20(d, 10.0)$ & 120.5 & $7.12(d, 10.0)$ \\
\hline $6 a$ & 133.5 & & 139.9 & & 139.2 & & 126.1 & & 124.3 & \\
\hline 7 & 188.0 & & 189.8 & & 189.9 & & 161.0 & & 156.7 & \\
\hline $7 a$ & 114.8 & & 115.2 & & 115.4 & & 112.9 & & 111.3 & \\
\hline 8 & 158.2 & & 158.9 & & 159.1 & & 187.6 & & 187.9 & \\
\hline $8-\mathrm{OH}$ & & $12.72(\mathrm{~s})$ & & & & $8.52(\mathrm{br} \mathrm{s})$ & & & & \\
\hline 9 & 137.1 & & 139.3 & & 139.7 & & 145.7 & & 144.5 & \\
\hline 10 & 133.7 & $7.90(d, 8.0)$ & 134.4 & $7.87(\mathrm{~d}, 8.0)$ & 134.4 & $7.93(d, 8.0)$ & 134.6 & $7.80(\mathrm{~s})$ & 135.8 & $8.13(\mathrm{~s})$ \\
\hline 11 & 119.7 & $7.71(\mathrm{dd}, 8.0,1.5)$ & 120.1 & $7.55(\mathrm{~d}, 8.0)$ & 120.1 & $7.64(d, 8.0)$ & 134.6 & & 127.7 & \\
\hline $11 a$ & 133.7 & & 132.0 & & 132.1 & & 117.5 & & 117.3 & \\
\hline 12 & 182.8 & & 183.5 & & 183.6 & & 141.6 & & 141.1 & \\
\hline $12 a$ & 135.9 & & 139.5 & & 140.4 & & 129.9 & & 129.4 & \\
\hline $12 \mathrm{~b}$ & 136.6 & & 78.9 & & 79.3 & & 80.4 & & 79.7 & \\
\hline \multicolumn{11}{|l|}{ 9-C-olivose } \\
\hline $1^{\prime}$ & 64.3 & $5.19(\mathrm{dd}, 11.5,2.0)$ & 65.5 & $5.19(\mathrm{dd}, 11.0,1.5)$ & 65.5 & $5.24(\mathrm{dd}, 7.5,1.5)$ & 66.0 & $5.03(\mathrm{~m})$ & 65.4 & $4.99(\mathrm{~d}, 11.0)$ \\
\hline $2^{\prime}$ & 39.5 & $\begin{array}{l}2.44 \text { (ddd, } 12.5,5.0,2.0 \text { ) } \\
1.40 \text { (q-like, } \sim 12.0 \text { ) }\end{array}$ & 41.0 & $\begin{array}{l}2.41(\mathrm{ddd}, 11.0,4.5,2.0) \\
1.34(\mathrm{~m})\end{array}$ & 41.1 & $\begin{array}{l}2.44(\mathrm{ddd}, 12.5,5.0,2.0) \\
1.37(\mathrm{~m})\end{array}$ & 40.7 & $\begin{array}{l}2.46(\mathrm{ddd}, 12.5,4.5,2.5) \\
1.30(\mathrm{~m})\end{array}$ & 41.0 & $\begin{array}{l}2.42(\mathrm{~m}) \\
1.30(\mathrm{~m})\end{array}$ \\
\hline $3^{\prime}$ & 68.4 & 4.03 (ddd, $11.0,9.5,5.0)$ & 68.8 & 3.96 (ddd, 11.0, 9.0, 5.5) & 68.8 & 3.97 (ddd, 11.0, 9.0, 5.0) & 68.7 & $3.94(\mathrm{~m})$ & 68.4 & 3.91 (ddd, $11.0,9.0,4.5)$ \\
\hline $4^{\prime}$ & 74.2 & $3.76(\mathrm{dd}, 9.0,6.0)$ & 74.9 & $3.66(d d, 9.0,5.5)$ & 75.0 & $3.66(d d, 9.0,6.0)$ & 74.7 & $3.55(\mathrm{dd}, 9.0,6.0)$ & 74.7 & $3.39(\mathrm{dd}, 9.0,4.5)$ \\
\hline $5^{\prime}$ & 72.6 & 4.44 (qui, 6.5) & 74.6 & 4.37 (qui, 6.5) & 74.6 & 4.38 (qui, 6.5) & 74.4 & 4.27 (qui, 6.5) & 73.6 & $4.05(\mathrm{~m})$ \\
\hline $6^{\prime}$ & 12.0 & $1.37(\mathrm{~d}, 7.0)$ & 12.5 & $1.37(\mathrm{~d}, 7.0)$ & 12.5 & $1.37(\mathrm{~d}, 7.0)$ & 12.6 & $1.30(\mathrm{~d}, 7.5)$ & 12.4 & $1.10(\mathrm{~d}, 6.5)$ \\
\hline \multicolumn{11}{|c|}{ 12-0-p-hydroxyphenylacetyl/12-0-indoleacetyl } \\
\hline $2^{\prime \prime}$ & & & & & & & 161.1 & & 159.6 & \\
\hline $3^{\prime \prime}$ & & & & & & & 133.0 & & 131.5 & \\
\hline $4^{\prime \prime}$ & & & & & & & 123.5 & & 109.2 & \\
\hline $5^{\prime \prime}$ & & & & & & & 134.6 & $7.36(d, 8.5)$ & 132.7 & $7.89(\mathrm{~s})$ \\
\hline $6^{\prime \prime}$ & & & & & & & 116.7 & $6.93(d, 8.5)$ & & \\
\hline $6 a^{\prime \prime}$ & & & & & & & & & 137.5 & \\
\hline $7^{\prime \prime}$ & & & & & & & 162.5 & & 113.1 & $7.55(d, 8.0)$ \\
\hline $8^{\prime \prime}$ & & & & & & & 116.7 & $6.93(d, 8.5)$ & 121.0 & $7.57(d, 8.0)$ \\
\hline $9^{\prime \prime}$ & & & & & & & 134.6 & $7.36(d, 8.5)$ & 121.6 & $7.14(d, 8.0)$ \\
\hline $10^{\prime \prime}$ & & & & & & & & & 123.6 & $7.23(d, 8.0)$ \\
\hline $10 a^{\prime \prime}$ & & & & & & & & & 128.3 & \\
\hline \multicolumn{11}{|c|}{ 4a-0-oliose/4a-0-rhodosamine } \\
\hline$r 1$ & & & 98.7 & $5.43(\mathrm{br} \mathrm{s})$ & 97.1 & $5.52(\mathrm{br} \mathrm{s})$ & 97.7 & 5.56 (br s) & 97.2 & $5.50(\mathrm{brd}, 2.0)$ \\
\hline r2 & & & 34.8 & $1.90(\mathrm{br} \mathrm{d}, 12.5)$ & 29.0 & $2.41(\mathrm{br} \mathrm{d}, 12.0)$ & 30.5 & $2.14(\mathrm{dd}, 12.0,2.5)$ & 30.3 & $1.98(\mathrm{dd}, 12.5,2.5)$ \\
\hline & & & & $1.84(\mathrm{~m})$ & & $2.00(\mathrm{~m})$ & & $1.84(\mathrm{td}, 12.5,3.5)$ & & $1.79(\mathrm{td}, 12.5,3.5)$ \\
\hline r3 & & & 66.7 & $3.98(\mathrm{~m})$ & 64.0 & $3.40(\mathrm{~m})$ & 63.4 & $2.35(\mathrm{~m})$ & 63.0 & $2.27(\mathrm{~m})$ \\
\hline r3- $N$-dimethyl & & & & & 42.1 & $3.03(\mathrm{br} \mathrm{s})$ & 43.9 & $2.36(\mathrm{~s})$ & 43.8 & $2.27(\mathrm{~s})$ \\
\hline $\mathrm{r} 4$ & & & 81.4 & $3.65(\mathrm{br} \mathrm{s})$ & 74.5 & $4.18(\mathrm{br} \mathrm{s})$ & 75.5 & $3.87(\mathrm{br} \mathrm{s})$ & 75.0 & 3.75 (br s) \\
\hline $\mathrm{r} 5$ & & & 68.3 & 4.13 (qui, 6.5) & 68.6 & 4.15 (qui, 6.5) & 69.4 & $4.09(q, 7.0)$ & 68.6 & $4.03(\mathrm{~m})$ \\
\hline r6 & & & 17.7 & $1.27(\mathrm{~d}, 6.5)$ & 18.3 & $1.35(\mathrm{~d}, 6.5)$ & 18.7 & $1.29(\mathrm{~d}, 5.5)$ & 18.4 & $1.22(\mathrm{~d}, 6.5)$ \\
\hline \multicolumn{11}{|l|}{ r4-0-oliose } \\
\hline 01 & & & 101.4 & $4.99(\mathrm{br} \mathrm{s})$ & 101.1 & $5.23(\mathrm{br} \mathrm{s})$ & 100.4 & 5.10 (t-like) & 100.0 & 4.93 (t-like) \\
\hline 02 & & & 33.2 & $1.95(\mathrm{td}, 11.7,4.0)$ & 33.1 & $\begin{array}{l}2.06(\mathrm{td}, 12.5,4.0) \\
1.98(\mathrm{~m})\end{array}$ & 33.3 & 1.95 (dd, 9.0, 3.0) & 33.6 & $1.83(\mathrm{~m})$ \\
\hline 03 & & & 66.6 & 4.07 (ddd, $11.7,4.0,2.5)$ & 66.3 & 4.05 (ddd, $11.5,4.0,2.5)$ & 66.8 & $4.08(\operatorname{td}, 7.5,3.0)$ & 66.2 & $3.98(\mathrm{~m})$ \\
\hline 04 & & & 72.3 & $3.61(\mathrm{~m})$ & 71.9 & $3.64(\mathrm{~m})$ & 72.5 & $3.58(\mathrm{br} \mathrm{d}, 2.0)$ & 72.1 & $3.50(\mathrm{br} \mathrm{d}, 2.0)$ \\
\hline 05 & & & 68.5 & 4.30 (qui, 6.5) & 68.9 & 4.13 (qui, 6.0) & 67.9 & $4.42(q, 6.5)$ & 67.2 & $4.46(q, 6.5)$ \\
\hline 06 & & & 17.2 & $1.23(\mathrm{~d}, 6.5)$ & 17.3 & $1.27(\mathrm{~d}, 6.5)$ & 17.4 & $1.23(\mathrm{~d}, 6.5)$ & 17.3 & $1.14(\mathrm{~d}, 6.5)$ \\
\hline
\end{tabular}

a-c Measured in $\mathrm{CDCl}_{3}, \mathrm{CD}_{3} \mathrm{OD}$ and $\mathrm{CD}_{3} \mathrm{COCD}_{3}$, respectively.

${ }^{d 13} \mathrm{C}$ and ${ }^{1} \mathrm{H}$ NMR were observed at 150 and $600 \mathrm{MHz}$, respectively.

${ }^{e}{ }^{13} \mathrm{C}$ and ${ }^{1} \mathrm{H}$ NMR were observed at 125 and $500 \mathrm{MHz}$, respectively. 


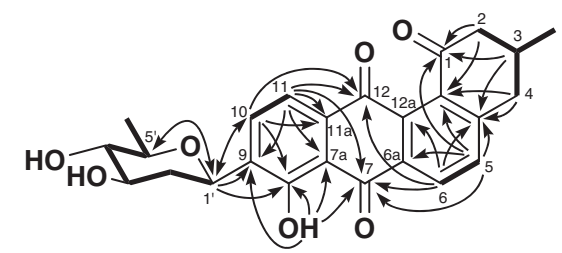

JBIR-90 (1)
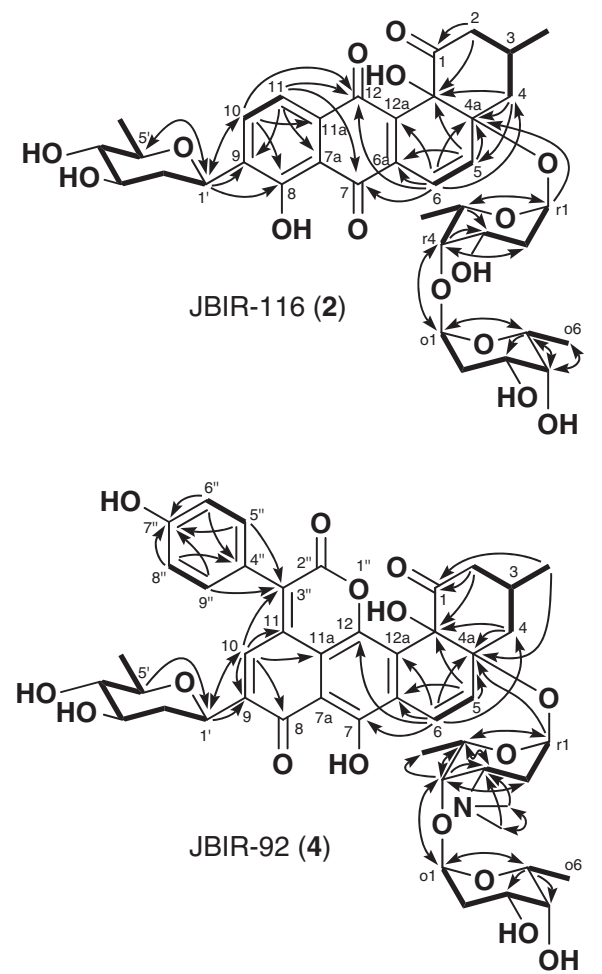
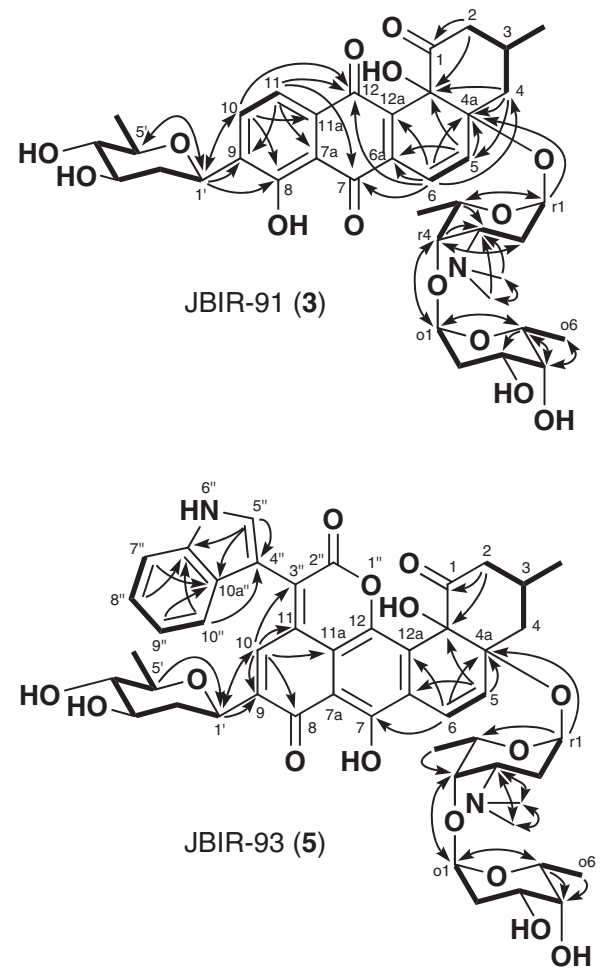

Figure 2 Key correlations of ${ }^{1} \mathrm{H}-{ }^{1} \mathrm{H}$ double-quantum-filtered COSY (bold lines) and constant time heteronuclear multiple bond correlation (solid arrows) of 1-5.

$\left(\delta_{\mathrm{H}} 1.01\right)$, and a coupling between two olefinic protons, $5-\mathrm{H}\left(\delta_{\mathrm{H}} 6.95\right)$ and 6-H $\left(\delta_{\mathrm{H}} 7.10\right)$, were observed in the DQF-COSY for 2 (Figure 2). The HMBC from $2-\mathrm{H}$ to a ketone carbonyl carbon C-1 $\left(\delta_{\mathrm{C}} 208.2\right)$ and an oxygenated quaternary carbon $\mathrm{C}-12 \mathrm{~b}\left(\delta_{\mathrm{C}} 78.9\right)$, from $4-\mathrm{H}$ to two oxygenated quaternary carbons, $\mathrm{C}-4 \mathrm{a}\left(\delta_{\mathrm{C}} 87.6\right)$ and $\mathrm{C}-12 \mathrm{~b}$, and an olefinic carbon C-5 $\left(\delta_{\mathrm{C}} 142.7\right)$, from $5-\mathrm{H}$ to $\mathrm{C}-4 \mathrm{a}$, an aromatic carbon $\mathrm{C}-6 \mathrm{a}\left(\delta_{\mathrm{C}} 139.9\right)$, and $\mathrm{C}-12 \mathrm{~b}$, and from $6-\mathrm{H}$ to $\mathrm{C}-4 \mathrm{a}$ and an aromatic carbon $\mathrm{C}-12 \mathrm{a}\left(\delta_{\mathrm{C}} 139.5\right)$ indicated the presence of a $3,4,4 \mathrm{a}, 8 \mathrm{a}-\mathrm{tetra}-$ hydro-4a,8a-dihydroxy-3-methyl-1(2H)-naphthalenone substructure. Other COSY and HMBC correlations were the same as those of $\mathbf{1}$, establishing an angucycline 9-C-olivoside structure, as shown in Figure 2. This 4a,12b-dihydroxyl form of angucycline was also supported by its UV absorption $\left(\lambda_{\max } 318\right.$ and $\left.425 \mathrm{~nm}\right) .{ }^{12,13}$ The presence of two additional sugar moieties was determined as follows. The sequences from the anomeric proton $\mathrm{r} 1-\mathrm{H}\left(\delta_{\mathrm{H}} 5.43\right)$ through the two methylene protons $\mathrm{r} 2-\mathrm{H}\left(\delta_{\mathrm{H}} 1.90,1.84\right)$ to an oxymethine proton $\mathrm{r} 3-\mathrm{H}\left(\delta_{\mathrm{H}} 3.98\right)$, and from an oxymethine proton $\mathrm{r} 4-\mathrm{H}\left(\delta_{\mathrm{H}} 3.65\right)$ through an oxymethine proton $\mathrm{r} 5-\mathrm{H}\left(\delta_{\mathrm{H}} 4.13\right)$ to a methyl proton r6$\mathrm{H}\left(\delta_{\mathrm{H}} 1.27\right)$, were observed in the DQF-COSY. Long-range couplings from $\mathrm{r} 1-\mathrm{H}$ to $\mathrm{C}-\mathrm{r} 5\left(\delta_{\mathrm{C}} 68.3\right)$ and $\mathrm{r} 4-\mathrm{H}$ to $\mathrm{C}-\mathrm{r} 3\left(\delta_{\mathrm{C}} 66.7\right)$ indicated a 2,6-dideoxyhexopyranoside structure. Their relative stereochemistry was determined by the coupling constants shown in Table 1, establishing an $\alpha$-2,6-dideoxy-lyxo-hexopyranoside (oliopyranoside) structure.
Another $\mathrm{O}$-glycoside unit, an $\alpha$-oliopyranoside, was deduced in the same manner, as shown in Figure 2. The ${ }^{1} \mathrm{H}-{ }^{13} \mathrm{C}$ long-range correlations from the two anomeric protons $\mathrm{r} 1-\mathrm{H}$ and o1- $\mathrm{H}\left(\delta_{\mathrm{H}} 4.99\right)$ to C$4 \mathrm{a}$ and $\mathrm{C}-\mathrm{r} 4\left(\delta_{\mathrm{C}} 81.4\right)$, respectively, concluded the substituted position of these glycosides, as shown in Figure 2. Thus, the structure of $\mathbf{2}$ was determined, as shown in Figure 1.

\section{Structure elucidation of 3}

Compound 3 was obtained as a dark yellow powder. The IR spectrum of 3 revealed the characteristic absorptions of a ketone ( $v_{\max }$ $\left.1720 \mathrm{~cm}^{-1}\right)$ and a quinone $\left(v_{\max } 1630 \mathrm{~cm}^{-1}\right)$ group. Its molecular formula was determined as $\mathrm{C}_{39} \mathrm{H}_{51} \mathrm{NO}_{14}$ by the HR-ESI-MS analysis. The ${ }^{13} \mathrm{C}$ and ${ }^{1} \mathrm{H}$ NMR spectral data for 3 (Table 1) revealed similar signals to those of 2 , except for the additional signal of an $\mathrm{N}$-dimethyl group. The long-range couplings from $\mathrm{r} 1-\mathrm{H}\left(\delta_{\mathrm{H}} 5.52\right)$ to $\mathrm{C}-\mathrm{r} 5\left(\delta_{\mathrm{C}}\right.$ 68.6), and from $\mathrm{r} 4-\mathrm{H}\left(\delta_{\mathrm{H}} 4.18\right)$ and an aminodimethyl proton $\mathrm{r} 3-\mathrm{N}$ $\mathrm{CH}_{3}\left(\delta_{\mathrm{H}} 3.03\right)$ to $\mathrm{C}-\mathrm{r} 3\left(\delta_{\mathrm{C}} 64.0\right)$ indicated a 3-(dimethylamino)-2,3,6trideoxyhexopyranoside moiety. Their relative stereochemistry was determined by the coupling constants to establish an $\alpha$-3-(dimethylamino)-2,3,6-trideoxy-lyxo-hexopyranoside (rhodosaminopyranoside) structure. Another $O$-glycoside unit, an $\alpha$-oliopyranoside, was deduced in the same manner, as shown in Figure 2. Thus, the structure of $\mathbf{3}$ was determined, as shown in Figure 1. 


\section{Structure elucidation of 4}

Compound 4 was obtained as a red powder and showed a protonated ion at $\mathrm{m} / z 890.3604$ (calcd. for $\mathrm{C}_{47} \mathrm{H}_{56} \mathrm{NO}_{16}, 890.3599$ ) in HR-ESIMS, corresponding to the molecular formula $\mathrm{C}_{47} \mathrm{H}_{55} \mathrm{NO}_{16}$. The IR spectrum $(\mathrm{KBr})$ of $\mathbf{4}$ revealed the characteristic absorptions of an ester and/or a ketone group $\left(v_{\max } 1720 \mathrm{~cm}^{-1}\right)$ and an unsaturated ketone functional group $\left(v_{\max } 1655,1640 \mathrm{~cm}^{-1}\right)$. The ${ }^{13} \mathrm{C}$ and ${ }^{1} \mathrm{H}$ NMR spectral data for 4 (Table 1) revealed similar signals to those of 3 and an additional six signals, including AB-type aromatic signals $\left(\mathrm{C}-5^{\prime \prime}, 9^{\prime \prime}\right.$, $\left.\delta_{\mathrm{C}} 134.6, \delta_{\mathrm{H}} 7.36 ; \mathrm{C}-6^{\prime \prime}, 8^{\prime \prime}, \delta_{\mathrm{C}} 116.7, \delta_{\mathrm{H}} 6.93\right)$ and an ester carbonyl signal $\left(\mathrm{C}-2^{\prime \prime}, \delta_{\mathrm{C}} 161.1\right)$.

The DQF-COSY and HMBC spectral analyses of 4 revealed that a $1,2,3,4,4 \mathrm{a}, 12 \mathrm{~b}$-hexahydrotetraphene, an $\alpha$-olivopyranoside, an $\alpha$-rhodosaminopyranoside and an $\alpha$-oliopyranoside moiety were conserved, as in 3 (Figure 2). The HMBC correlations from the AB-type aromatic proton $5^{\prime \prime}, 9^{\prime \prime}-\mathrm{H}$ to a phenolic carbon $\mathrm{C}-7^{\prime \prime}\left(\delta_{\mathrm{C}} 162.5\right)$ and an aromatic carbon $\mathrm{C}-3^{\prime \prime}\left(\delta_{\mathrm{C}}\right.$ 133.0) at the peri-position, and from another AB-type aromatic proton $6^{\prime \prime}, 8^{\prime \prime}-\mathrm{H}$ to an aromatic quaternary carbon $\mathrm{C}-4^{\prime \prime}\left(\delta_{\mathrm{C}} 123.5\right)$ revealed the phenol moiety. Long-range couplings from $10-\mathrm{H}\left(\delta_{\mathrm{H}} 7.80\right)$ to $\mathrm{C}-3^{\prime \prime}$ suggested that the phenol moiety and the tetraphene unit are attached through $\mathrm{C}-3^{\prime \prime}$. A remaining unit, an ester carbonyl carbon C-2" $\left(\delta_{\mathrm{C}} 161.1\right)$, was found to form a six-membered ring system consisting of C-2", C- $3^{\prime \prime}, \mathrm{C}-11, \mathrm{C}-11 \mathrm{a}, \mathrm{C}-12$ and an oxygen atom, as shown in Figure 2. This characteristic chromophore was supported by UV and visible absorption data $\left(\lambda_{\max } 310\right.$ and $504 \mathrm{~nm}$ ) in comparison with those of urdamycin C. ${ }^{12,14}$ Thus, the structure of $\mathbf{4}$ was determined as a $p$-hydroxyphenylacetic acid complex of 3, as shown in Figure 1.

\section{Structure elucidation of 5}

Compound $\mathbf{5}$ was obtained as a violet powder, and its HR-ESI-MS $(\mathrm{m} /$ $z$ 911.3621; calcd. for $\mathrm{C}_{49} \mathrm{H}_{55} \mathrm{~N}_{2} \mathrm{O}_{15}, 911.3602$ ) indicated the molecular formula $\mathrm{C}_{49} \mathrm{H}_{56} \mathrm{~N}_{2} \mathrm{O}_{15}$. The IR spectrum (KBr) of 5 revealed the characteristic absorptions of an ester and/or a ketone group ( $v_{\max }$ $\left.1720 \mathrm{~cm}^{-1}\right)$ and an unsaturated ketone functional group $\left(v_{\max } 1630\right.$, $1600 \mathrm{~cm}^{-1}$ ). The ${ }^{13} \mathrm{C}$ and ${ }^{1} \mathrm{H}$ NMR spectral data for 5 (Table 1) revealed that the signals in the condensed ring unit and the sugar units were super-imposable with those of 4 . The structural differences between $\mathbf{4}$ and $\mathbf{5}$ are as follows.

The sequence from an aromatic proton $7^{\prime \prime}-\mathrm{H}\left(\delta_{\mathrm{H}} 7.55\right)$ through two aromatic protons, $8^{\prime \prime}-\mathrm{H}\left(\delta_{\mathrm{H}} 7.57\right)$ and $9^{\prime \prime}-\mathrm{H}\left(\delta_{\mathrm{H}} 7.14\right)$, to an aromatic proton $10^{\prime \prime}-\mathrm{H}\left(\delta_{\mathrm{H}} 7.23\right)$ was observed in the DQF-COSY (Figure 2). ${ }^{1} \mathrm{H}-{ }^{13} \mathrm{C}$ long-range couplings from an aromatic proton $5^{\prime \prime}-\mathrm{H}\left(\delta_{\mathrm{H}} 7.89\right)$ to three aromatic carbons, C- $4^{\prime \prime}\left(\delta_{\mathrm{C}} 109.2\right), \mathrm{C}-6 \mathrm{a}^{\prime \prime}\left(\delta_{\mathrm{C}} 137.5\right)$ and C$10 \mathrm{a}^{\prime \prime}\left(\delta_{\mathrm{C}} 128.3\right)$, and a low-field shifted ${ }^{13} \mathrm{C}$ chemical shift of C-5" $\left(\delta_{\mathrm{C}}\right.$ 132.7) and C- $6 \mathrm{a}^{\prime \prime}$ indicated the presence of an indole structure. The UV and visible absorption spectra of $5\left(\lambda_{\max } 328\right.$ and $\left.578 \mathrm{~nm}\right)$ were the same as those of the known indoleacetic acid-conjugated angucycline urdamycin D. ${ }^{12,14}$ Thus, the structure of 5 was determined as a $3^{\prime \prime}-(3-$ indolyl)-substituted congener of 4 instead of the hydroxyphenyl group, as shown in Figure 1.

\section{Cytotoxicity}

The cytotoxic effects of $\mathbf{1}-\mathbf{5}$ on MPM and HeLa cells were determined by the WST-8 (2-(2-methoxy-4-nitrophenyl)-3-(4-nitrophenyl)-5-(2,4-disulfophenyl)- $2 \mathrm{H}$-tetrazolium monosodium salt) colorimetric assay. Compounds 2, 3 and 4 exhibited cytotoxic activities against ACC-MESO-1 cells, with $\mathrm{IC}_{50}$ values of 45,23 and $46 \mu \mathrm{M}$, respectively (Table 2). However, $\mathbf{1}$ and $\mathbf{5}$ did not show cytotoxicity against ACC-MESO-1 cells at a concentration of $100 \mu \mathrm{M}$. In addition, $\mathbf{1 - 5}$ exhibited cytotoxic activity against HeLa cells with $\mathrm{IC}_{50}$ values of $18-75 \mu \mathrm{m}$ (Table 2).
Table 2 Cytotoxic effects of 1-5 on MPM and HeLa cells

\begin{tabular}{lrcccr}
\hline & \multicolumn{5}{c}{ CC $_{50}(\mu \mathrm{M})$} \\
\cline { 2 - 6 } Cancer cell lines & \multicolumn{1}{c}{$\mathbf{1}$} & $\mathbf{2}$ & $\mathbf{3}$ & $\mathbf{4}$ & \multicolumn{1}{c}{$\mathbf{5}$} \\
\hline ACC-MESO-1 & 100 & 45 & 23 & 46 & $>100$ \\
HeLa & 47 & 23 & 18 & 18 & 75 \\
\hline
\end{tabular}

\section{METHODS}

\section{General experimental procedures}

Optical rotations were obtained on an SEPA-300 polarimeter (Horiba, Kyoto, Japan). UV and IR spectra were measured on a DU730 UV/Vis spectrophotometer (Beckman Coulter, Brea, CA, USA) and an FT-720 spectrophotometer (Horiba), respectively. NMR spectra were recorded on a Varian NMR System 500 or $600 \mathrm{NB}$ CL (Varian, Palo Alto, CA, USA) in $\mathrm{CDCl}_{3}$ (7.25 p.p.m. for ${ }^{1} \mathrm{H}, 77.0$ p.p.m. for ${ }^{13} \mathrm{C}$ ) or $\mathrm{CD}_{3} \mathrm{OD}$ (3.35 p.p.m. for ${ }^{1} \mathrm{H}, 49.0$ p.p.m. for $\left.{ }^{13} \mathrm{C}\right)$ or acetone- $d_{6}\left(2.04\right.$ p.p.m. for ${ }^{1} \mathrm{H}, 29.8$ p.p.m. for ${ }^{13} \mathrm{C}$ ), with the residual solvent peak as the internal standard. HR-ESI-MS data were recorded on an LCT Premier XE mass spectrometer (Waters, Milford, MA, USA). Normal-phase medium-pressure liquid chromatography was performed using a Purif-Pack SI 30 column $(30 \mu \mathrm{m}$; Shoko Scientific, Yokohama, Japan). Analytical reversedphase HPLC was carried out using a Capcell Pak C18 MGII column $(5.0 \mu \mathrm{m}$, $4.6 \mathrm{~mm}$ i.d. $\times 150 \mathrm{~mm}$; Shiseido, Tokyo, Japan) equipped with a 2996 photodiode array detector (Waters) and a 3100 Mass Detector (Waters). Preparative reversed-phase HPLC was carried out using a Capcell Pak C18 MGII column (5.0 $\mu \mathrm{m}, 20 \mathrm{~mm}$ i.d. $\times 150 \mathrm{~mm}$; Shiseido).

\section{Microorganisms}

The producing Streptomyces designated as RI33 was isolated from a lichen obtained from a soil sample collected at Shuri (Okinawa Prefecture, Japan) by the SDS-yeast extract method. ${ }^{15}$ The genus of the strain RI33 was identified with the partial $16 \mathrm{~S}$ rRNA gene sequences $(660 \mathrm{bp})$ by using EzTaxzon. ${ }^{16}$ The strain belonged to the genus Streptomyces, as the $16 \mathrm{~S}$ rRNA gene sequence comparison exhibited high sequence similarity of $99.24 \%$ to that of Streptomyces albiaxialis strain NRRL B-24327 ${ }^{\mathrm{T}}$ (accession no. AY999901).

\section{Fermentation}

Streptomyces sp. RI33 was cultivated in 50-ml test tubes each containing $15 \mathrm{ml}$ of a seed medium consisting of starch $1 \%$ (Kosokagaku, Tokyo, Japan), Polypepton 1\% (Nihon Pharmaceutical, Tokyo, Japan), molasses 1\% (DaiNippon Meiji Sugar, Tokyo, Japan) and meat extract 1\% (Extract Ehrlich; Wako Pure Chemical Industries, Osaka, Japan), pH 7.2 (before sterilization). The test tubes were shaken on a reciprocal shaker ( 355 r.p.m.) at $27^{\circ} \mathrm{C}$ for 2 days. Aliquots $(2.5 \mathrm{ml})$ of the broths were transferred to $500-\mathrm{ml}$ baffled Erlenmeyer flasks (20 flasks) containing $100 \mathrm{ml}$ of a production medium consisting of starch 2.5\% (Kosokagaku), soybean meal 1.5\% (Nisshin Oillio Group, Tokyo, Japan), dry yeast $0.2 \%$ (Mitsubishi Tanabe Pharma, Osaka, Japan), $\mathrm{CaCO}_{3}$ 0.4\% (Kozakai Pharmaceutical, Tokyo, Japan) and Diaion HP-20 1\% (Mitsubishi Chemical, Tokyo, Japan), pH 7.2 (before sterilization), and cultured on a rotary shaker ( 180 r.p.m.) at $27^{\circ} \mathrm{C}$ for 5 days.

\section{Isolation}

The mycelial cake obtained from the fermentation broth (21) was extracted with $\mathrm{Me}_{2} \mathrm{CO}(500 \mathrm{ml})$. The extract was concentrated in vacuo, and the aqueous concentrate was extracted with EtOAc $(100 \mathrm{ml} \times 3)$. After drying over anhydrous $\mathrm{Na}_{2} \mathrm{SO}_{4}$, the organic layer was evaporated to dryness. The residue $(845 \mathrm{mg})$ was subjected to normal-phase medium-pressure liquid chromatography and successively eluted with a stepwise solvent system of $n$-hexaneEtOAc (0, 10, 20 and 25\% EtOAc) and $\mathrm{CHCl}_{3}-\mathrm{MeOH}(0,2,5,10,20$ and 50\% $\mathrm{MeOH})$. The $5 \% \mathrm{MeOH}$ fraction $(54.7 \mathrm{mg})$ was purified by preparative reversed-phase HPLC with $65 \% \mathrm{MeOH}-\mathrm{H}_{2} \mathrm{O}$ (flow rate $10 \mathrm{ml} \mathrm{min}^{-1}$ ) to yield 1 (6.3 mg, Rt $24.8 \mathrm{~min})$. The $10 \% \mathrm{MeOH}$ fraction $(31.4 \mathrm{mg}$ ) was purified by preparative reversed-phase HPLC with $55 \% \mathrm{MeOH}-\mathrm{H}_{2} \mathrm{O}$ (flow rate 
$\left.10 \mathrm{ml} \mathrm{min}^{-1}\right)$ to yield $2(4.0 \mathrm{mg}$, Rt $18.8 \mathrm{~min})$. The $20 \% \mathrm{MeOH}$ fraction (31.4 mg) was purified by preparative reversed-phase HPLC with a $35-70 \%$ $\mathrm{MeOH}-\mathrm{H}_{2} \mathrm{O}$ linear gradient system $\left(30 \mathrm{~min}\right.$, flow rate $\left.10 \mathrm{ml} \mathrm{min}^{-1}\right)$ to yield 3 (12.6 mg, Rt $23.4 \mathrm{~min})$. The $50 \% \mathrm{MeOH}$ fraction $(27.1 \mathrm{mg}$ ) was subjected to preparative reversed-phase HPLC with a $50-90 \% \mathrm{MeOH}-\mathrm{H}_{2} \mathrm{O}$ linear gradient system $(30 \mathrm{~min})$ followed by a $90 \% \mathrm{MeOH}(10 \mathrm{~min})$ isocratic system to yield two fractions ( $4.1 \mathrm{mg}$, Rt $26.8 \mathrm{~min}$ and $5.9 \mathrm{mg}$, Rt $30.9 \mathrm{~min}$ ) containing 4 and $\mathbf{5}$, respectively. From the former fraction (Rt $26.8 \mathrm{~min}), \mathbf{4}(0.9 \mathrm{mg})$ was isolated by a Toyopearl HW-40F gel filtration column $\left(15 \times 500 \mathrm{~mm}^{2}\right.$; Tosoh, Tokyo, Japan) developed with $100 \% \mathrm{MeOH}$. From the latter fraction (Rt $30.9 \mathrm{~min}$ ), 5 ( $1.3 \mathrm{mg}$, Rt $13.8 \mathrm{~min})$ was obtained by using preparative reversed-phase HPLC with a linear gradient system $\left(35-90 \% \mathrm{MeOH}-\mathrm{H}_{2} \mathrm{O}, 20 \mathrm{~min}\right.$, flow rate $10 \mathrm{ml} \mathrm{min}^{-1}$ ). The physicochemical properties of compounds thus obtained are described below.

JBIR-90 (1). Yellow powder; $[\alpha]_{\mathrm{D}}{ }^{25}+86.3(c 0.15, \mathrm{MeOH}) ; \mathrm{UV}(\mathrm{MeOH}) \lambda_{\max }$ (ع) 268 (22 200) and $405(4100) \mathrm{nm}$; IR (KBr) $v_{\max } 1705$ and $1620 \mathrm{~cm}^{-1} ;{ }^{1} \mathrm{H}$ NMR $\left(600 \mathrm{MHz}, \mathrm{CDCl}_{3}\right)$ and ${ }^{13} \mathrm{C} \mathrm{NMR}\left(150 \mathrm{MHz}, \mathrm{CDCl}_{3}\right)$, see Table 1; HRESI-MS $m / z 437.1592[\mathrm{M}+\mathrm{H}]^{+}$(calcd. for $\mathrm{C}_{25} \mathrm{H}_{25} \mathrm{O}_{7}, 437.1600$ ).

JBIR-116 (2). Dark yellow powder; optical rotation could be not measured by the yellow sodium D line near $589 \mathrm{~nm}$; UV $(\mathrm{MeOH}) \lambda_{\max }(\varepsilon) 318(5400)$ and 425 (6500) nm; IR (KBr) $v_{\max } 1720$ and $1640 \mathrm{~cm}^{-1} ;{ }^{1} \mathrm{H}$ NMR $(500 \mathrm{MHz}$, $\left.\mathrm{CD}_{3} \mathrm{OD}\right)$ and ${ }^{13} \mathrm{C}$ NMR $\left(125 \mathrm{MHz}, \mathrm{CD}_{3} \mathrm{OD}\right)$, see Table 1; HR-ESI-MS $\mathrm{m} / \mathrm{z}$ $729.2750[\mathrm{M}-\mathrm{H}]^{-}$(calcd. for $\mathrm{C}_{37} \mathrm{H}_{45} \mathrm{O}_{15}, 729.2758$ ).

JBIR-91 (3). Dark yellow powder; optical rotation could be not measured; $\mathrm{UV}(\mathrm{MeOH}) \lambda_{\max }(\varepsilon) 319(5600)$ and $423(6800) \mathrm{nm}$; IR (KBr) $v_{\max } 1720$ and $1630 \mathrm{~cm}^{-1} ;{ }^{1} \mathrm{H}$ NMR $\left(600 \mathrm{MHz}, \mathrm{CD}_{3} \mathrm{OD}\right)$ and ${ }^{13} \mathrm{C}$ NMR $\left(150 \mathrm{MHz}, \mathrm{CD}_{3} \mathrm{OD}\right)$, see Table 1; HR-ESI-MS $\mathrm{m} / z 758.3375[\mathrm{M}+\mathrm{H}]^{+}$(calcd. for $\mathrm{C}_{39} \mathrm{H}_{52} \mathrm{NO}_{14}$, 758.3388).

JBIR-92 (4). Red powder; optical rotation could be not measured; UV $(\mathrm{MeOH}) \lambda_{\max }(\varepsilon) 310(15900)$ and $504(11100) \mathrm{nm}$; IR (KBr) $v_{\max } 1720$, 1655 and $1640 \mathrm{~cm}^{-1} ;{ }^{1} \mathrm{H}$ NMR $\left(500 \mathrm{MHz}, \mathrm{CD}_{3} \mathrm{OD}\right)$ and ${ }^{13} \mathrm{C} \mathrm{NMR}(125 \mathrm{MHz}$, $\mathrm{CD}_{3} \mathrm{OD}$ ), see Table 1; HR-ESI-MS $\mathrm{m} / \mathrm{z} 890.3604 \quad[\mathrm{M}+\mathrm{H}]^{+}$(calcd. for $\left.\mathrm{C}_{47} \mathrm{H}_{56} \mathrm{NO}_{16}, 890.3599\right)$.

JBIR-93 (5). Violet powder; optical rotation could be not measured; UV $(\mathrm{MeOH}) \lambda_{\max }(\varepsilon) 328(11900)$ and $578(10400) \mathrm{nm}$; IR $(\mathrm{KBr}) v_{\max } 1720,1630$ and $1600 \mathrm{~cm}^{-1}$; ${ }^{1} \mathrm{H}$ NMR $\left(500 \mathrm{MHz}, \mathrm{CD}_{3} \mathrm{COCD}_{3}\right)$ and ${ }^{13} \mathrm{C} \mathrm{NMR}(125 \mathrm{MHz}$, $\mathrm{CD}_{3} \mathrm{COCD}_{3}$ ), see Table 1; HR-ESI-MS $\mathrm{m} / \mathrm{z} 911.3621[\mathrm{M}-\mathrm{H}]^{-}$(calcd. for $\mathrm{C}_{49} \mathrm{H}_{55} \mathrm{~N}_{2} \mathrm{O}_{15}, 911.3602$ ).

\section{Cytotoxic assay}

Cytotoxic activities against human MPM ACC-MESO- 1 cells $^{17}$ and human cervical carcinoma HeLa cells were determined by a colorimetric assay, using WST-8. ACC-MESO-1 and HeLa cells were cultured in RPMI 1640 (Nacalai Tesque, Kyoto, Japan) and Dulbecco's modified Eagle's medium (Sigma, St Louis, MO, USA), respectively, supplemented with $10 \%\left(\mathrm{vv}^{-1}\right)$ fetal bovine serum (Life Technologies, Carlsbad, CA, USA), penicillin $\left(100 \mathrm{Uml}^{-1}\right)$ and streptomycin $\left(100 \mu \mathrm{g} \mathrm{ml}^{-1}\right)$ at $37^{\circ} \mathrm{C}$ in a humidified incubator under a $5 \%$ $\mathrm{CO}_{2}$ atmosphere. The 384-well plates were seeded with aliquots of a $20-\mu \mathrm{l}$ medium containing $1 \times 10^{3}$ cells per well and were incubated overnight before being treated with compounds at various concentrations for $48 \mathrm{~h}$. Plates were incubated for $1 \mathrm{~h}$ at $37^{\circ} \mathrm{C}$ after the addition of $2 \mu \mathrm{l}$ of WST- 8 reagent solution (Cell Counting Kit; Dojindo, Kumamoto, Japan) per well. The absorption of the formed formazan dye was measured at $450 \mathrm{~nm}$. The vehicle solvent (DMSO) was used as a negative control. We screened all the samples at a final concentration of $1 \%$ and selected a sample that showed $\geqslant 70 \%$ inhibition of the growth of ACC-MESO-1 and HeLa cells.

\section{ACKNOWLEDGEMENTS}

This work was supported by a grant from the New Energy and Industrial Technology Development Organization (NEDO), Japan and a Grant-in-Aid for Scientific Research (20380070 to KS) from the Japan Society for the Promotion of Science (JSPS).

1 Carbone, M., Kratzke, R. A. \& Testa, J. R. The pathogenesis of mesothelioma. Semin. Oncol. 29, 2-17 (2002).

2 Mossman, B. T., Kamp, D. W. \& Weitzman, S. A. Mechanisms of carcinogenesis and clinical features of asbestos-associated cancers. Cancer Invest. 14, 466-480 (1996).

3 Robinson, B. W. S. \& Lake, R. A. Advances in malignant mesothelioma. N. Engl. J. Med. 353, 1591-1603 (2005).

4 Tsiouris, A. \& Walesby, R. K. Malignant pleural mesothelioma: current concepts in treatment. Nat. Clin. Pract. Oncol. 4, 344-352 (2007).

5 Weder, W. et al. Neoadjuvant chemotherapy followed by extrapleural pneumonectomy in malignant pleural mesothelioma. J. Clin. Oncol. 22, 3451-3457 (2004).

6 Motohashi, K., Hwang, J.- H., Sekido, Y., Takagi, M. \& Shin-ya, K. JBIR-23 and -24, novel anticancer agents from Streptomyces sp. AK-AB27. Org. Lett. 11, 285-288 (2009).

7 Hwang, J.- H., Takagi, M. Murakami, H., Sekido, Y \& Shin-ya, K. Induction of tubulin polymerization and apoptosis in malignant mesothelioma cells by a new compound JBIR-23. Cancer Lett. 300, 189-196 (2011).

8 Izumikawa, M., Khan, S. T., Komaki, H., Takagi, M. \& Shin-ya, K. JBIR-31, a new teleocidin analog, produced by salt-requiring Streptomyces sp. NBRC 105896 isolated from a marine sponge. J. Antibiot. 63, 33-36 (2010).

9 Ueda, J., Takagi, M. \& Shin-ya, K. Aminocaprophenone- and pyrrolidine-type alkaloids from the leaves of Ficus septica. J. Nat. Prod. 72, 2181-2183 (2009).

10 Motohashi, K., Takagi, M., Yamamura, H., Hayakawa, M. \& Shin-ya, K. A new angucycline and a new butenolide isolated from lichen-derived Streptomyces spp. J. Antibiot. 63, 545-548 (2010).

11 Furihata, K. \& Seto, H. Constant time HMBC (CT-HMBC), a new HMBC technique useful for improving separation of cross peaks. Tetrahedron Lett. 39, 7337-7340 (1998).

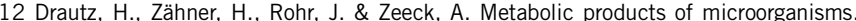
234. Urdamycins, new angucycline antibiotics from Streptomyces fradiae. I. Isolation, characterization and biological properties. J. Antibiot. 39, 1657-1669 (1986).

13 Rohr, J. \& Zeeck, A. Metabolic products of microorganisms. 240. Urdamycins, new angucycline antibiotics from Streptomyces fradiae. II. Structural studies of urdamycins B to F. J. Antibiot. 40, 459-467 (1987).

14 Rohr, J., Zeeck, A. \& Floss, H. G. Urdamycins, new angucycline antibiotics from Streptomyces fradiae. III. The structures of urdamycins C and D. J. Antibiot. 41, 126-129 (1988)

15 Hayakawa, M. \& Nonomura, H. A new method for the intensive isolation of actinomycetes from soil. Actinomycetologica 3, 95-104 (1989).

16 Chun, J. et al. EzTaxon: a web-based tool for the identification of prokaryotes based on $16 \mathrm{~S}$ ribosomal RNA gene sequences. Int. J. Syst. Evol. Microbiol. 57, 2259-2261 (2007).

17 Usami, N. et al. Establishment and characterization of four malignant pleural mesothelioma cell lines from Japanese patients. Cancer Sci. 97, 387-394 (2006). 